\title{
A Quasi-Static Cluster-Computing Approach for Dynamic Channel Assignment in Cellular Mobile Communication Systems
}

\author{
YU-KWONG KWOK \\ Department of Electrical and Electronic Engineering \\ The University of Hong Kong, Pokfulam Road, Hong Kong \\ Email: ykwok@eee.hku.hk
}

\begin{abstract}
Efficient management of the radio spectrum can be accomplished by making use of channel assignment techniques, which work by allocating different channels of the spectrum to the cells of the network in a conflict-free manner (i.e., the cochannel interference is minimized). The problem of dynamically reallocating the channels in response to change in user location patterns, which occurs frequently for a micro-cell network architecture, is even more difficult to tackle in a timely manner. Most existing approaches use various sequential search based heuristics which cannot produce high quality allocation fast enough to cope with the frequent traffic requirement variations. In this paper, we propose a quasi-static approach which combines the merits of both static and dynamic schemes. The static component of our approach uses a parallel genetic algorithm to generate a suite of representative assignments based on a set of different estimated traffic scenarios. At on-line time, the dynamic component observes the actual traffic requirement and retrieve the representative assignment of the closest scenario from the off-line table. The retrieved assignment is then quickly refined by using a fast parallel local search algorithm. Our extensive simulation experiments have indicated that the proposed quasi-static system outperforms other dynamic channel assignment techniques significantly in terms of both blocking probabilities and computational overhead.
\end{abstract}

Keywords: Cellular communications, dynamic channel assignment, genetic algorithms, local search, parallel algorithms, cluster computing.

\section{INTRODUCTION}

Recently, we have witnessed a spectacular growth in the use of cellular mobile communication systems. Although advances in wireless communication technologies have enabled a dramatic increase in network bandwidth, developers also keep on coming up with applications that use more bandwidth. Thus, it is still important to efficiently utilize the nonetheless scarce radio spectrum using a proper channel assignment scheme [6]. Specifically, the objective of a channel assignment algorithm is to determine a spectrumefficient allocation of channels to the cells such that the

$\uparrow$ This work was supported by a grant from the HKU CRCG. traffic demand can be satisfied as far as possible under the channel reuse constraints. A channel can be reused in that the same channel can be assigned to multiple cells simultaneously due to the radio propagation path loss. However, there are three types of channel reuse constraints: co-channel constraints, channels separation constraints, and co-site constraints [6], [12]. The channel assignment problem has been tackled by many researchers in the last two decades [6]. In a broad sense, channel assignment algorithms can be classified as static and dynamic. In a static approach, also commonly called fixed channel assignment (FCA) [12], the channel allocation pattern is carefully determined off-line and will not be changed even when traffic requirements vary. In a dynamic approach, usually referred to as dynamic channel assignment (DCA) [14], channels are allocated to the cells dynamically depending upon the current traffic demand. The major merit of FCA over DCA is that FCA, being a static technique, can afford to spend more time to come up with a better solution and is also easier to implement in practice. However, a major weakness of FCA is that its solutions highly depend on the input traffic scenarios, which may deviate considerably at on-line time. In addition, FCA is too inflexible to cope with the frequent traffic requirement variations in nowadays microcell-based networks. Obviously, from a resource utilization point of view, DCA is more preferable to FCA because DCA is designed to adjust resource allocation according to traffic demand, and hence, can support a higher capacity (or lower blocking probability). The demerits of a DCA scheme are its algorithm efficiency and ease of implementation. Thus, devising a DCA, that is practical, efficient, and can generate high quality allocations, is a challenging problem. In this paper, we attempt to meet this challenge by proposing a new DCA system based on a novel approach.

There have been many approaches proposed for the DCA problem [6]. Since the DCA problem is shown to be NP-hard, heuristic approaches are usually sought. These approaches usually use search algorithms based on evolutionary or local search techniques such as neural networks [2], genetic algorithms [12], combinatorial evolution schemes [13], simulated annealing [5], and Tabu search [1]. However, these schemes generally 
suffer from high complexity and, thus, may not be suitable to be used in practice. As discussed above, we observe that a pure static or dynamic approach is not suitable. In our study, we have designed and implemented a hybrid channel allocation system that combines the merits of both static and dynamic approaches. In view of the computational complexity of the problem, we use a parallel approach which can be executed on a cluster-computing system-a network of workstations or PCs. Advances in microprocessor and network technologies have enabled such kinds of computing platforms to be highly cost-effective and easily accessible. In addition to enhanced efficiency, a parallel approach can potentially generate better solutions than those of a sequential approach, as demonstrated in [8], [9]. The proposed allocation system consists of two parts: an off-line module and an on-line module, which are both based on parallel search methods. The off-line module uses a parallel genetic algorithm (PGA) to generate a set of allocation patterns corresponding to different user mobility scenarios sampled from a space induced by expected traffic requirements. The on-line module will then, based on the current scenario, retrieve the most suitable allocation pattern, which will be dynamically refined using a very efficient parallel local search (PLS) algorithm based on the hybrid channel borrowing and reassignment strategies. Such a table-lookup followed by parallel refinement approach is faster than other DCA schemes while it can potentially generate better solutions. Indeed, the proposed system has been tested extensively using a cluster of workstations (connected by an $155 \mathrm{Mbps}$ Fore ATM switch) and is found to be superior to other DCA schemes both in terms of solution quality (i.e., blocking probability) and efficiency. The system we have implemented is as shown in Figure 1.

This paper is organized as follows. Section 2 contains an outline of our proposed DCA system. Section 3 provides some highlights of our experimental results. The last section concludes the paper

\section{A QUaSi-STatic APproach Using a CLUSTER OF WORKSTATIONS}

Similar to other related approaches [12], [13], we assume that the cellular radio network consists of $m$ arbitrarily connected cells. The traffic demands of the cells, in terms of number of channels, are represented by

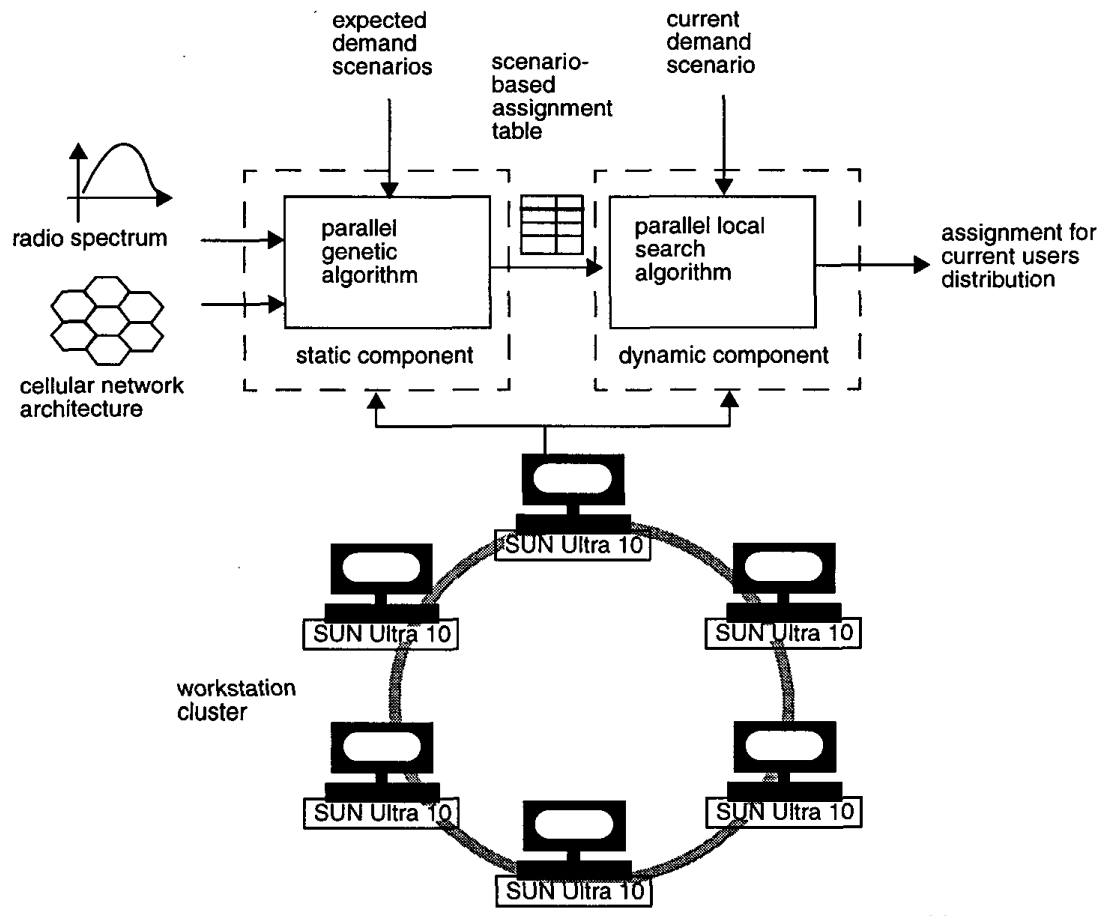

Figure 1: A cluster-computing based approach to the dynamic channel assignment problem. 
an $m$-element demand vector $D$, where each element $d_{i}$ is the number of channels required for cell $i$. On the other hand, we assume there are $n$ channels given. To specify an assignment, we use a binary variable $f_{i j}$ such that if $f_{i j}=1$ means channel $j$ is assigned to cell $i$; otherwise, $f_{i j}=0$. To satisfy the demand requirement, we must have $\sum_{j=1}^{n} f_{i j} \geq d_{i}$ for all $i$. In addition, the reuse constraints are represented by a compatibility matrix $C$, where $c_{i i}$ is the minimum separation distance between any two channels in cell $i$, and $c_{i k}$ is the minimum frequency separation between any two frequencies assigned to cells $i$ and $k$. The objective of the assignment algorithm is to determine a valid allocation pattern that can minimize the expected blocking probability. Notice that this is different from the goal of minimizing the frequency span [4].

In the static component of our proposed DCA system, we use a genetic algorithm (GA) in which a chromosome is defined as the string of bits $f_{i j}$. Mutation is simply a random flipping of a randomly selected $f_{i j}$. We have implemented three different crossover operators: the order crossover, the partially-mapped (PMX) crossover, and the cyclic crossover [8]. Most importantly, a parallel GA (PGA) is used in our system [8] and, therefore, high quality assignments can be determined efficiently using a workstation cluster. In our quasi-static approach, it is assumed that the ranges of the dynamic parameters are known. This assumption is justified because, for example, for a particular size of user population, the maximum possible number of calls is bounded and can be estimated. Once specified by the wireless network administrator, the space of dynamic demand vectors (DDV), of which the boundaries are determined by the ranges, is partitioned into a certain number of disjoint regions. Within each region, a certain number of random dynamic demand vectors are chosen. The off-line PGA is then applied to determine the mappings for these sample scenarios represented by different dynamic parameter vectors. Afterwards, the mapping for each sample scenario is exhaustively evaluated for every other sample scenario in the region by applying the assignment to the network with the corresponding traffic demand and computing the expected blocking probability. The assignment that gives the smallest average blocking probability is chosen as the representative assignment for the corresponding region in the dynamic demand space, and is stored in the offline assignment table in the form of an indexed multidimensional array. The static component is schematically depicted in Figure 2.

The input to the on-line module will consist of an actual distribution of calls (i.e., the actual demand vector). We model the changing traffic requirements as a sequence of different varying demand vectors. We call such a sequence a traffic profile (TP). Each TP consists of $t$ iterations of demand vectors $D^{r}(0 \leq r \leq t-1)$ such that $D^{r+1}=D^{r}+\Delta^{r}$ where each element $\delta_{i}$ of $\Delta^{r}$ is the change in traffic requirement in cell $i$. Thus, when iteration $r$ begins, the on-line module does not know the actual values of the dynamic demand vector $D^{r}$ for that iteration. Invoked periodically with a period of $\tau$ units of time, the on-line module has to determine an assignment for iteration $r$ based on the dynamic demand vector of iteration $(r-1)$. In the semi-static mapping approach, given the dynamic vector of iteration $(r-1)$, the on-line module retrieves the assignment corresponding to the representative dynamic demand vector closest to the given actual values (closest is on a per cell requirement

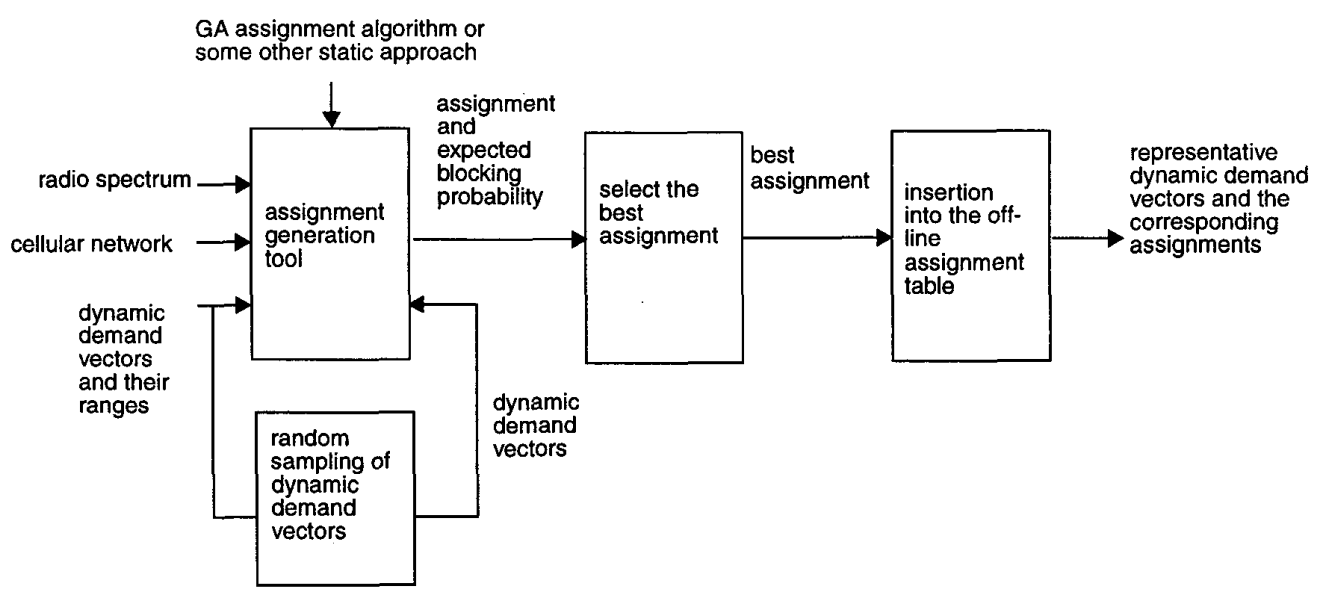

Figure 2: Generation of off-line assignments. 
basis). The retrieved assignment is then further refined using a very fast parallel local search algorithm [9]. The refining procedure is targeted for two goals: (1) the number of reallocations which require physically redistribute the channels; and (2) the expected blocking probability which is determined by using the input traffic demand pattern (new call and handoff requests). Notice that such the input traffic pattern is essentially a piece of history data which may not reflect the current traffic load. However, with a properly tuned value of $\tau$, we can reasonably expect that the new traffic pattern will be similar to the input pattern. Obviously, there is a tradeoff between quality of re-assignment and the computational overhead (for determining the new assignment and for physically deploying it) involved. The refinement is done by probabilistically swapping If the refined pre-determined assignment is expected to give a smaller blocking probability, a redistribution of channels is performed; otherwise, the assignment used at iteration $(r-1)$ will continue to be used for iteration $r$. Notice that at a microcell level, a hybrid channel borrowing strategy is employed to determined the number of required channels. More details of the search algorithms can be found in [7].

\section{RESULTS}

We have performed extensive simulation experiments to evaluate the proposed quasi-static scheme. The simulation environment is similar to those considered in [3], [11], [14]. The simulated cellular network has 49 regular hexagonal cells. We assume a Poisson arrival process with rate $\mu_{N}$ for new call requests in each cell. The call durations are exponentially distributed with mean $1 / \alpha_{T}$. In addition, the time period between a new call request and a handoff request is assumed to be exponentially distributed with mean $\mu_{H}$. All these stochastic processes are assumed to be independent. Using a discrete event-driven simulator, we ran each experiment for a duration of $10^{8}$ events. In each experiment, the system started with a uniformly distributed random traffic pattern. The confidence interval of each simulation is $95 \%$.

We compared the quasi-static (abbreviated as QS hereafter) with several other DCA schemes as well as a FCA algorithm. However, for brevity, we include below only the results of the comparison with the borrowing with directional channel locking (BDCL) scheme [14] and the uniform fixed channel assignment method [10]. Detailed results can be found in [7]. Basically, we observed the new call blocking probability and the handoff request blocking probability of the three schemes in the experiments. These probabilities were computed overall the whole simulation period for all cells in each experiment. These results are shown in
Figure 3(a) and (b). As can be seen, the two DCA schemes outperform the FCA scheme by a large margin. The proposed QS method consistently gives lower blocking probabilities for new call and handoff requests. As in [3], we also computed the reallocation ratio of the two DCA methods, which is defined as the total number of re-assignments divided by the total number of new calls. This performance metric reflects the computational efficiency of the DCA method in that the higher the reallocation ratio, the more time (and power) should be used to redistribute the channels. We can see from Figure 3(c) that the QS scheme is far better than the BDCL method due to the fact that the QS scheme uses an efficient local search step to minimize the number of reassignments necessary to achieve a lower expected blocking probability, while the BDCL method performs reassignment without regard to the physical overhead involved. Furthermore, the aggregate computation time required to adapt to traffic demand changes in the QS scheme is also much lower than that in the BDCL method because the on-line component of the QS scheme is only invoked periodically.

\section{CONCLUSIONS AND FUTURE WORK}

We address the dynamic channel assignment (DCA) problem for cellular mobile systems. We have outlined our proposed quasi-static (QS) approach for the DCA problem. The proposed approach, which consists of a parallel genetic algorithm as the static component and a parallel local search algorithm as the dynamic component, has been implemented on a cluster of twelve Sun Ultra workstations connected by a high speed ATM switch. The DCA system has been evaluated using extensive simulations and is found to outperform other DCA schemes significantly in terms of blocking probabilities and computational overhead. The proposed quasi-static system can be executed using a network of workstations or PCs so that it can be a practicable and scalable approach for efficient allocation of channels in a real environment. Further work is underway to investigate the effect of the invocation period of the online component on the performance of the QS DCA scheme [7].

\section{References}

[1] D.J. Castelino, S. Hurley, and N.M. Stephens, "A Tabu Search Algorithm for Frequency Assignment," Annals of Operations Research, vol. 63, pp. 301-319, 1996.

[2] P.T.H. Chan, M. Palaniswami, and D. Everitt, "Neural Network-Based Dynamic Channel Assignment for Cellular Mobile Communication Systems," IEEE Trans. Vehicular Technology, vol. 43, no. 2, pp. 279-288, May 1994.

[3] K.-N. Chang, J.-T. Kim, C.-S. Yim, and S. Kim, "An Efficient Borrowing Channel Assignment Scheme for Cellular Mobile Systems," IEEE Trans. Vehicular Technology, vol. 47, no. 2, pp. 602-608, May 1998. 


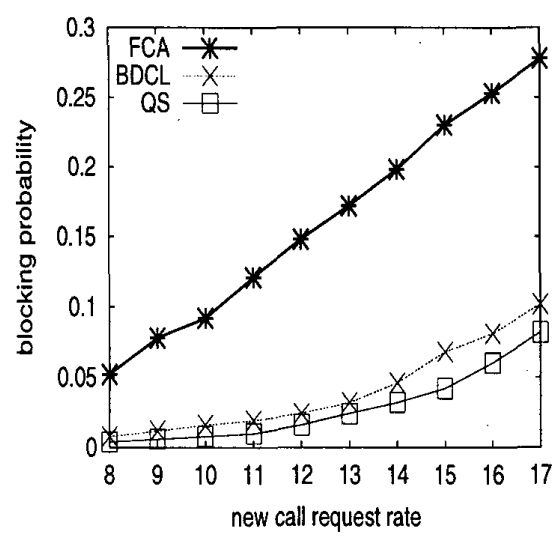

(a) new call blocking probability

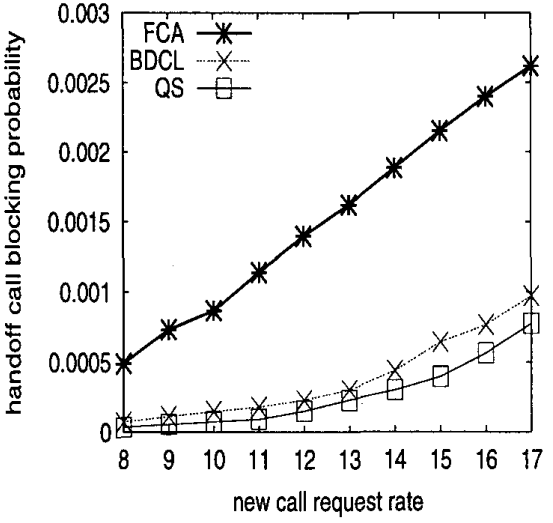

(b) handoff call blocking probability

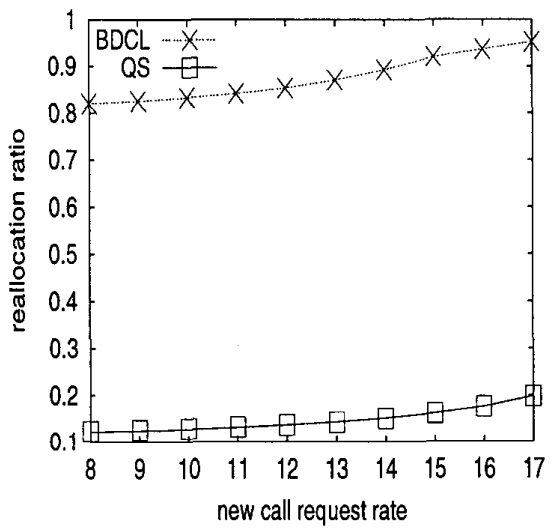

(c) reallocation ratio

Figure 3: Performance of the proposed quasi-static (QS) scheme compared to a DCA (BDCL) and a FCA approach.

[4] J.C.-I Chuang "Performance Issues and Algorithms for Dynamic Channel Assignment," IEEE J. Selected Areas in Communications, vol. 11 , no. 6, pp. 955-963, Aug. 1993.

[5] M. Duque-Anton, D. Kunz, and B. Ruber, "Channel Assignment for Cellular Radio using Simulated Annealing," IEEE Trans. Vehicular Technology, vol. 42, no. 1, pp. 14-21, Feb. 1993.

[6] I. Katzela and M. Naghshineh, "Channel Assignment Schemes for Cellular Mobile Telecommunication Systems: A Comprehensive Survey," IEEE Personal Communications, vol. 3, no. 6, pp. 10-31, June 1996.

[7] Y.-K. Kwok, "A Novel Quasi-Static Scheme for Dynamic Channel Assignment for Cellular Mobile Systems," in preparation.

[8] Y.-K. Kwok and I. Ahmad, "Efficient Scheduling of Arbitrary Task Graphs to Multiprocessors Using A Parallel Genetic Algorithm," J. Parallel and Distributed Computing, vol. 47, no. 1, pp. 58-77, Nov. 1997.

[9] Y.-K. Kwok and I. Ahmad, "FASTEST: A Practical Low Complexity Algorithm for Compile-Time Assignment of Parallel Programs to Multiprocessors," IEEE Trans.
Parallel and Distributed Systems, vol. 10, no. 2, pp. 147159, Feb. 1999.

[10] W.C.Y. Lee, Mobile Cellular Telecommunications Systems, New York: McGraw-Hill, 1989.

[11] J. Li, N.B. Shroff, and E.K.P. Chong, "Channel Carrying: A Novel Handoff Scheme for Mobile Cellular Networks," IEEE/ACM Trans. Networking, vol. 7, no. 1, pp. 38-50, Feb. 1999.

[12] C.Y. Ngo and V.O.K. Li, "Fixed Channel Assignment in Cellular Radio Networks Using a Modified Genetic Algorithm," IEEE Trans. Vehicular Technology, vol. 47, no. 1, pp. 163-172, Feb. 1998.

[13] H.G. Sandalidis, P.P. Stavroulakis, and J. RodriguezTellez, "An Efficient Evolutionary Algorithm for Channel Resource Management in Cellular Mobile Systems," IEEE Trans. Evolutionary Computation, vol. 2, no. 4, pp. 125-137, Nov. 1998

[14] M. Zhang and T.-S. P. Yum, "Comparisons of ChannelAssignment Strategies in Cellular Mobile Telephone Systems," IEEE Trans. Vehicular Technology, vol. 38, no. 3, pp. 211-215, Nov. 1989. 